Research article

\title{
Counting the costs: Comparing depot medroxyprogesterone acetate and norethisterone oenanthate utilisation patterns in South Africa

\author{
Jennifer Smit ${ }^{* 1,2,3}$, Andrew Gray ${ }^{2}$, Lynn McFadyen ${ }^{1,2,3}$ and \\ Khangelani Zuma ${ }^{4}$
}

\begin{abstract}
Address: ${ }^{1}$ Africa Centre for Population Studies and Reproductive Health, Mtubatuba, South Africa, ${ }^{2}$ Pharmaceutical Policy Practice Group, School of Pharmacy and Pharmacology, University of Durban-Westville, Durban, South Africa, ${ }^{3}$ Division of HIV Prevention and Vaccine Research, South African Medical Research Council, Durban, South Africa and ${ }^{4}$ Biostatistics Unit, South African Medical Research Council, Durban, South Africa

E-mail: Jennifer Smit* - jensmit@mweb.co.za; Andrew Gray - andy@ healthlink.org.za; Lynn McFadyen - lmcfadyen@mrc.ac.za; Khangelani Zuma - kz2@Waikato.ac.nz

*Corresponding author
\end{abstract}

This article is available from: http://www.biomedcentral.com/1472-6963/I/4

(c) 200 I Smit et al, licensee BioMed Central Ltd.

\begin{abstract}
Background: In South Africa, where health care resources are limited, it is important to ensure that drugs provision and use is rational. The Essential Drug List includes depot medroxyprogesterone acetate (DMPA) and norethisterone oenanthate (NET-EN) as injectable progestagen-only contraceptives (IPCs), and both products are extensively used.
\end{abstract}

Objectives and Methods: Utilisation patterns of the injectable contraceptive products DMPA and NET-EN are compared in the context of current knowledge of the safety and efficacy of these agents. Utilisation patterns were analysed by means of a Pareto (ABC) analysis of IPCs issued from 4 South African provincial pharmaceutical depots over 3 financial years. A case study from rural KwaZulu-Natal, South Africa, is used to examine utilisation patterns and self-reported side effects experienced by 187 women using IPCs.

Results: IPCs accounted for a substantial share of total state expenditure on drugs. While more DMPA than NET-EN was issued, NET-EN distribution from 2 depots increased over the 3-year period. Since DMPA was cheaper, if all NET-EN clients in the 1999/2000 financial year (annualised) had used DMPA, the 4 depots could have saved 4.95 million South African Rands on product acquisition costs alone. The KZN case study showed slightly more NET-EN (54\%) than DMPA $(46 \%)$ use; no significant differences in self-reported side effects; and that younger women were more likely to use NET-EN than DMPA $(p=0.0001)$.

Conclusions: Providing IPCs on the basis of age is not appropriate or cost effective. Rational use of these products should include consideration of the cost of prescribing one over another.

Introduction

Affordability of drugs by developing countries is current- ly a topic of heated debate. In South Africa, where financial resources for health care are limited, and where 
health care costs are expected to soar as the HIV epidemic escalates, it becomes increasingly important to ensure that all drugs are rationally provided and used. The injectable progestagen-only contraceptives (IPCs) depot medroxyprogesterone acetate (DMPA) and norethisterone oenanthate (NET-EN) are by far the most widely utilised contraceptives in South Africa, especially amongst younger users and women living in rural areas [1]. Both drugs are on the South African Essential Drug List [2] and are available free of charge at public sector primary health care facilities. Although not extensively documented, it is claimed that there has been a shift away from the predominant use of DMPA, which is given every 12 weeks, to NET-EN, given every 8 weeks, especially amongst younger, nulliparous women [3, 4]. Combined injectable contraceptives (CICs), which contain a combination of oestrogen and progestagen, are not registered for use in South Africa.

The World Health Organisation's general criteria - safety, affordability, necessity and efficacy - for inclusion on the Model List of Essential Drugs (EDL) [5] provide a useful basis upon which to make decisions about drug selection and rational use. Taking into account published findings on efficacy, reversibility, side effects and safety, this paper analyses IPC supply patterns and costs from four pharmaceutical depots, and describes a case study of IPC utilisation patterns and side effects in a rural subdistrict of KwaZulu-Natal, South Africa. Based on these analyses, appropriate recommendations for the rational use of IPCs are made.

\section{What the literature tells us}

Published clinical trials and reviews on efficacy, side effects, reversibility and safety of DMPA and NET-EN were sought by means of computerized and hand searches. Copies of relevant publications and citations from these publications were obtained and reviewed. Relevant international and South African policy documents were also reviewed. This extensive search revealed that: DMPA is better researched than NET-EN, few studies directly compare DMPA and NET-EN, few clinical trials have been undertaken in Southern Africa, few clinical studies have been undertaken amongst young users, and most published studies, upon which review after review are based, were undertaken in the 1970 s and early 1980 s. Methodological differences in subject recruitment, exclusion criteria, frequency and nature of procedures for follow-up, types of observations made, method of recording, methods of analysis and large intersite variability in some studies, make it difficult to evaluate the published data. Trussel et al. provide a useful account of difficulties in analysing and comparing contraceptive efficacy trials [6]. Nevertheless, to the extent that this is possible, a comparative synopsis of the efficacy, side ef- fects, delay in return to fertility and safety of DMPA and NET-EN is provided in this section. It is not the purpose of this paper to provide a detailed review, but merely to highlight relevant findings. The authors can be contacted for a more extensive bibliography.

Both IPCs are demonstrably highly effective. There are minor differences in published efficacy rates of both drugs depending on the study, timing of the first injection, the population, body weight, dosage regimen and provider training. An illustration of the high efficacy of these two products is provided by a World Health Organisation (WHO) comparative trial [7]. According to this study the efficacy of DMPA given every 90 days and NET-EN given every 60 days are comparable, with a cumulative 2-year pregnancy rate of 0.4 per 100 womanyears. In an evaluation of 5 large controlled multicentre studies, Kaunitz [8] reported that there were only 24 pregnancies among 7849 women using DMPA for 122 496 patient-months. Trussel et al. [6] provide "summary estimates of contraceptive failure" and give the lowest expected, and typical percentage, of accidental pregnancies in the United States, during the first year of use, as 0.3 for DMPA and 0.4 for NET-EN (unspecified dose interval).

There is little direct comparative data on the reversibility of DMPA and NET-EN. While return to fertility is reported by some reviewers to be more rapid with NET-EN [9, 10], more recently, Bigrigg et al. [11] in examining early data, suggest that there is no delay in return to fertility with DMPA use, if one considers the methodological bias of early studies, which did not take in to account the date of the last DMPA injection. They state further that "if there is a delay it is not statistically significant and is less than 30 days". Kaunitz gives the shortest reported time before fertility is returned with DMPA, as 4 months after the last injection i.e. 4 weeks after the due date of the next injection [8] and, according to Hatcher et al. return to fertility is delayed by DMPA for about 4 months longer on average, compared with the combined oral contraceptive method, intrauterine contraceptive device, and condoms [12].

The poor side effect profile of progestagen-only injectables is extensively documented. The most frequently reported side effects, and those most likely to result in discontinuation, are menstrual disturbances such as amenorrhoea, irregular bleeding and heavy bleeding [3, 13]. Menstrual irregularities are reported to occur more often with DMPA than with NET-EN use. For instance, the WHO clinical trial undertaken in 1983 compared menstrual disturbances resulting from DMPA given at 90-day intervals, with NET-EN given every 60 days and with NET-EN given every 60 days for 6 months and then 
every 84 days [7]. Significantly less amenorrhoea was reported by NET-EN users (on both dosage regimens), than by DMPA users. Amenorrhoea was also found to result in significantly higher discontinuation rates with DMPA users than with NET-EN. During the first six months of use, both dosage regimens of NET-EN were reported to result in more defined cyclic patterns and fewer prolonged bleeding and spotting episodes than DMPA, but similar discontinuation rates were found with the two products. However, in a study undertaken in Egypt, despite the more frequent occurrence of menstrual irregularities with DMPA, better one-year continuation rates were found with DMPA than with NET-EN [14]. Weight gain is also a commonly reported side effect and in comparing DMPA and NET-EN, the findings on weight gain appear to be similar. A multinational WHO comparative clinical trial found no statistical difference in weight gain between NET-EN and DMPA (both administered at 12 week intervals) after a year of use - the weight gain with NET-EN was reported as $1.5 \mathrm{~kg}$ and with DMPA was $2.0 \mathrm{~kg}$ [15]. Headache was the most common non-menstrual side effect reported in this comparative trial and was more frequently reported by DMPA users than NET-EN users, however, it is important to note that in this study, NET-EN was administered every 12 weeks.

IPCs are considered to be relatively safe contraceptive methods $[16,17]$ and recent studies indicate that there is little reason to be concerned about either DMPA or NETEN causing an increased risk of breast cancer [18]. However, the possible effect of DMPA on bone density, particularly in adolescents and long-term users is cause for concern [19]. Little is published on the possible effect of NET-EN on bone density. Findings from prospective studies in progress are awaited.

The World Health Organization's Medical Eligibility Criteria for Contraceptive Use classifies DMPA and NET-EN together, and makes no differentiation between the two in regard to their side effects or contraindications [20]. The only restriction this document makes about age, for IPC use, is that "For women under 16 years of age, there are theoretical concerns regarding hypooestrogenic effects..." p.54. The WHO states further that there is no need to restrict use of progestagen-only contraceptive methods for nulliparous women. The Primary Health Care Essential Drugs List for South Africa provides no guidelines with respect to the circumstances under which DMPA rather than NET-EN should be prescribed (or vice versa) [2].

\section{Methods}

\section{Supply patterns and costs}

Consumption figures of IPC stock issued from provincial pharmaceutical depots were requested from the Deputy Director, Procurement of the South African National Department of Health. Data for DMPA and NET-EN were made available for the KwaZulu-Natal (KZN), Guateng and Free State Provincial Pharmaceutical depots and for the Port Elizabeth depot, which serves the western part of the Eastern Cape Province. These four provinces (of nine South African provinces) represent over 50\% of the total South African population. Gauteng has a mostly urban population and KZN and Eastern Cape are more rural. The following data were analysed for financial years $1997 / 8,1998 / 9$, and for $1 / 04 / 99$ to $7 / 12 / 99$ of the $1999 /$ 2000 financial year:

- Position number on Pareto (ABC) analyses for DMPA and NET-EN. An ABC analysis is a method which ranks drugs according to their annual usage (unit cost times annual consumption). Class A items are the 10 to $20 \%$ which account for 75 to $80 \%$ of the funds spent. Class B items have an intermediate contribution to total expenditure, whereas Class $\mathrm{C}$ items (the majority of items) account for a small percentage of funds spent. $\mathrm{ABC}$ analyses are used to identify priority cost drivers for intervention [21].

- Number of units of each item issued in the same time period per depot.

- Total cost of each item per time period per depot (at constant 1999 prices).

- Current and previous tender prices for DMPA and NET-EN. Note exchange rate: 1 British Pound $\approx 11$ South African Rands.

\section{Case study: use patterns and side effects}

Prevalence of IPC use was determined by means of a community-based cross-sectional survey undertaken in a rural sub-district in northern KZN, South Africa. Commencing from a randomly selected starting point, every second household was chosen until $40 \%$ of households in the sub-district had been visited. In this way, 849 households of an estimated 2088 were selected and, one woman from each household, in the age range 15 to 49, was randomly selected for interview. Verbal and written explanations of the study were provided to each woman selected (in Zulu and/or English) and consent to participate in the study was requested. In all, 848 women were interviewed and no-one refused to participate. Prior to commencing the survey, workshops and meetings were held to introduce the study to local traditional leaders, community health workers, and health service pro- 
viders. Ethical clearance for the study was provided by the Ethics Committee of the University of Durban-Westville.

Each woman selected was asked if she was currently using an IPC and those who were, were asked whether they were using DMPA or NET-EN. Data were collected by means of an extensive structured interview, including questions on demographic characteristics, reasons for method selection, and problems and side effects experienced. Interviews were conducted in Zulu, between September and December 1998. They were conducted during the day from Mondays to Fridays, but where a selected woman was not home, a revisit was made in the evening or on a Saturday. Data were coded, double entered and analysed using Epi-Info Version 6.43 and the SAS Version 6.12.

Table I: Acquisition costs of injectable contraceptive products: 1997/8, 1998/9, 1999/2000

\begin{tabular}{llll}
\hline Product & Cost per vial (SAR ${ }^{*}$ ) & & $1999 / 2000$ \\
& $1997 / 8$ & $1998 / 9$ & \\
DMPA & & 4.56 & 4.78 \\
$\begin{array}{l}\text { Innovator product (Pharmacia Upjohn) } \\
\text { Generic product (Aspen Pharmacare) }\end{array}$ & 2.17 & 2.07 & 4.29 \\
\hline $\begin{array}{l}\text { NET-EN } \\
\text { Innovator product (Schering) }\end{array}$ & $-\#$ & & 4.78 \\
\end{tabular}

*Exchange rate: I British Pound $\approx$ II South African Rands (SAR) \# Tender not awarded

Table 2: Pareto analysis of injectable contraceptive products: 1997/8, 1998/9, 1999/2000

\begin{tabular}{|c|c|c|c|}
\hline \multirow[t]{2}{*}{ Pharmaceutical Depot } & \multicolumn{3}{|c|}{ Rank } \\
\hline & $1997 / 8$ & $1998 / 9$ & $1999 / 2000$ \\
\hline \multicolumn{4}{|l|}{ GAUTENG } \\
\hline DMPA & 3 & 4 & 4 \\
\hline NET-EN & 2 & 3 & 2 \\
\hline \multicolumn{4}{|l|}{ KWAZULU-NATAL } \\
\hline DMPA & 4 & 6 & 4 \\
\hline NET-EN & 19 & 19 & 19 \\
\hline \multicolumn{4}{|l|}{ FREE SATE } \\
\hline DMPA & 2 & 3 & 5 \\
\hline NET-EN & 5 & 5 & 6 \\
\hline \multicolumn{4}{|l|}{ PORT ELIZABETH } \\
\hline DMPA & 3 & 6 & 3 \\
\hline NET-EN & 4 & 4 & 4 \\
\hline
\end{tabular}




\section{Results}

\section{Supply patterns and costs}

\section{Cost of injectable contraceptive products}

DMPA products issued at primary health care outlets in the three financial years analysed were obtained from the original patent holder (Pharmacia-Upjohn) and a generic manufacturer (Aspen Pharmacare). NET-EN was available only from the innovator (Schering). Table 1 shows that the acquisition cost of a vial of both DMPA and NET-EN products increased every year, and that the cost of both DMPA products rose particularly steeply. In the 1999/2000 financial year, the generic product, was almost the same price as the innovator product. Their costs were exactly the same in the 1999/2000 fiscal year.

Since DMPA is given less frequently than NET-EN, cost per couple years of protection (CYP) provides a more accurate cost comparison of DMPA and NET-EN. Based on the 1999/2000 state tender prices for the DMPA and NET-EN innovator products, the cost per couple year was SAR28.68 for NET-EN (6 vials per year) and SAR19.12 for DMPA (4 vials per year). If the calculation were based on the DMPA generic product price, use of DMPA would be even cheaper (SAR17.16). It should be noted that the cost of syringes, needles and swabs, personnel costs and client transport and time were not included in the calculations. These costs can be considerable and are obviously higher for NET-EN because it is administered more frequently.

\section{Analysis of annual expenditure on DMPA and NET-EN}

In all 4 depots, both IPCs consumed an important share of total drug expenditure (table 2). A Pareto analysis shows that both DMPA and NET-EN appeared in the top 10 in each year (based on actual volumes multiplied by constant 1999 prices), with the exception of NET-EN in KZN where it was19 ${ }^{\text {th }}$ in $1997 / 8,1998 / 9$ and $1999 / 2000$. More was spent on NET-EN than DMPA in Gauteng in all 3 years, but less in the other 3 depots. Only in 1998/9 in the Port Elizabeth area was more spent on NET-EN than DMPA. Total annualised expenditure on both products in the 4 depots in $1999 / 2000$ was projected to be SAR28.77 million.

\section{Ratio of NET-EN:DMPA issued}

The ratios of NET-EN:DMPA issued from the 4 depots were calculated based on CYP rather than on number of vials issued. As shown in Figure 1, DMPA was increasingly used in Port Elizabeth where the ratio of NET-EN:DMPA decreased from 0.64 in 1997/8 to 0.57 in 1999/200o. In Free State the market share was more or less stable (0.42, 0.44, 0.40). A similar picture emerged in KZN (o.22, 0.23, 0.25), with some increase in NET-EN use. However, in Gauteng, while DMPA was still used most, NET-EN use was clearly increasing $(0.67,0.73,0.81)$.

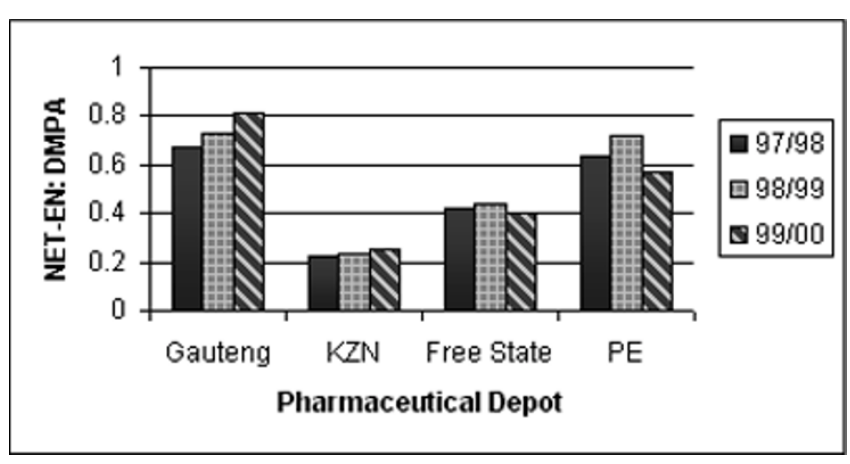

Figure I

Ratio of NET-EN:DMPA issued from the four pharmaceutical depots in 1997/8, 1998/9, 1999/2000

Counting the cost of injectable contraceptive product choice If all NET-EN clients in the 1999/2000 financial year (annualised) had been given DMPA instead, the 4 depots together might have saved SAR4.95 million. Conversely, if NET-EN had been issued to all DMPA clients, then the estimated additional cost in the same year for the 4 depots would have been SAR9.35 million. Savings and additional costs would be increased if other costs (surgical supplies, personnel costs, client transport etc.) were included. The savings are calculated on the annualised total CYP for innovator versions of both DMPA and NETEN. If the price of the cheaper generic preparation of DMPA had been used in the calculation, the savings would have been greater.

To illustrate the potential savings or additional costs further, in KZN, a 9.0\% saving on the expected 1999/2000 annual IPC drug bill might have been effected if only DMPA had been supplied. On the other hand, if only NET-EN had been available, the additional load would have been $36.5 \%$. In Gauteng, the savings or additional costs could have been $18.3 \%$ and $22.6 \%$ respectively.

\section{Case study: use patterns and side effects}

Injectable contraceptive prevalence and cost

All respondents (848) were African, Zulu-speaking women in the age range 15-49 years. Of these, 187 (22.1\%) were using an injectable contraceptive method, either the innovator product of DMPA or NET-EN. Forty-six per cent (86) of the IPC users were using DMPA and 54\% (101) were using NET-EN. The mean age of DMPA users was 29.6 years (median 29, range 18-49) and that of NET-EN users was 23.2 (median 23; range 17-37). Younger women were thus more likely to use NET-EN than DMPA ( $\mathrm{p}=0.0001)$. The age distribution of DMPA and NET-EN users is shown in Figure 2. The mean length of use was 2.2 years (range 0.1 to 11 ). 


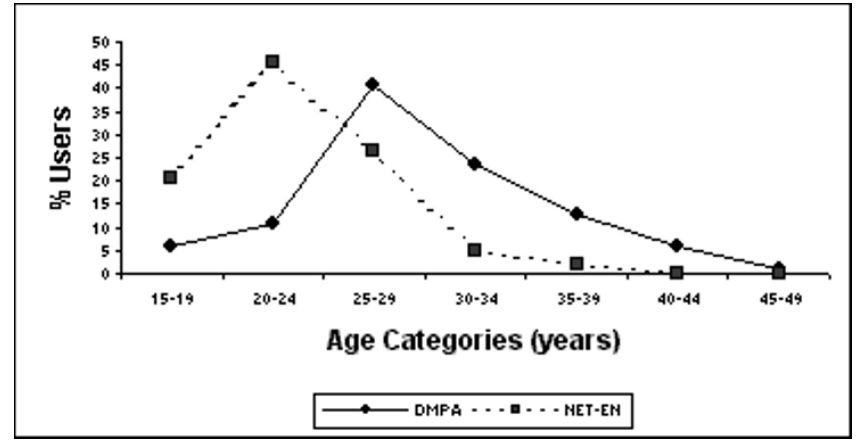

Figure 2

Age distribution of DMPA and NET-EN users

The ratio of NET-EN:DMPA users in this rural sub-district was 1.2 to 1 . Based on 1999/2000 product costs, by supplying only DMPA, a saving of $21.3 \%$ on the annual drug bill for IPCs could have been achieved by the local health facility. On the other hand, if only NET-EN had been supplied, the annual IPC cost would have increased by $18.1 \%$. These figures are based on product costs alone.

\section{Reasons for product choice}

Current users were asked, by means of an open-ended question, why they preferred the injectable product they were using, and the following findings are of note:

- Many DMPA users (42.4\%) indicated that they preferred this product because it was "stronger". On the other hand, NET-EN was favoured by $36.0 \%$ of those using it as it was regarded as "weaker" or "lighter".

- Concern about delayed return to fertility with DMPA was expressed by $5.0 \%$ of NET-EN users and $14.0 \%$ indicated that they chose NET-EN because it did not delay return to fertility.

- The idea that the NET-EN is for younger women or teenagers and DMPA for older women was expressed by $14.6 \%$ of the IPC users. This preference is clearly reflected in the age distribution of DMPA \& NET-EN users depicted in Figure 2.

- Recommendation by health worker was given as one of the most common reasons for product choice (21.1\%).

- Relatively few women (6.5\%) mentioned that concern about side effects influenced choice of either product.

\section{Side effects with injectable contraceptives}

IPC users were asked to indicate what side effects, if any, they were experiencing with DMPA or NET-EN by responding to a list of 22 possible side effects. Consistent with the international literature, many women reported menstrual irregularities such as amenorrhoea, spotting, heavy periods or irregular periods (table 3). Other side effects commonly reported were vaginal wetness and weight gain. The side effect profile for DMPA or NET-EN users was similar with no significant differences found between users of the two products in terms of their experience of side effects.

\section{Discussion}

In highlighting key issues in financing family planning services in Sub-Saharan Africa, Janowitz et al. make the following statement "Given limited resources, the universal provision of methods based on demand and without regard to cost will restrict the number of individuals whose need for family planning services can be met" $\mathrm{p}$. 64 [22]. The balancing of needs and resources becomes even more challenging when attempting to meet reproductive health needs more broadly. For instance, in developing countries like South Africa many drugs, such as antiretrovirals for the management of HIV, are not available through the public sector. Careful analysis of current expenditure on drugs is thus required so that resources are allocated to meet changing therapeutic needs.

Findings presented in this paper show that IPCs account for a substantial share of the total state expenditure on drugs in South Africa. Of the two IPCs available on the EDL, DMPA is a cheaper option than NET-EN, even if only considering acquisition costs. Analysis of supply patterns from the 4 pharmaceutical depots shows that if all NET-EN clients had been given DMPA, between 9.0\% and $18.3 \%$ of the expected annual drug bill for IPCs could have been saved per depot. Rational use of drugs cannot however be based on cost alone and clinical criteria, such as efficacy, safety, and acceptability of side effects, must also be considered. The context within which contraception is provided should also be taken into account. An extensive review of the literature on IPCs shows little difference between NET-EN and DMPA in terms of efficacy, safety, reversibility and side effect profile. However, a systematic comparative review has not been published and little clinical data on African women are available. NET-EN appears to have a slightly better side effect profile and a slightly shorter delay in return to fertility. DMPA is marginally more effective and is more convenient as users only have to return to the clinic every 12 weeks rather than after 8 weeks, as is the case for NET-EN users. As noted earlier, no differentiation in regard to side effects or contraindications of the two products is made by the WHO in terms of its medical eligibility criteria for contraceptive use [20].

Findings from the KZN case study show that slightly more NET-EN was used than DMPA. No significant dif- 
ferences were found in self-reported experience of side effects. What does emerge clearly is that NET-EN is viewed as the product of choice for young women and DMPA for older women. This is reflected in reasons given for product preference by clients, and in the age distribution of DMPA and NET-EN users. Further, health workers appear to play an important role in decisionmaking about which IPC product is provided. That different products are considered to be more appropriate for different age groups may be linked to the perception that DMPA is "stronger" while NET-EN is "weaker", and may well be related to concerns about delay in return to fertility after IPC use, particularly with DMPA. This is consistent with results from a study undertaken in the Northern Province of South Africa where providers were found to recommend NET-EN for younger women based on their perception that DMPA use may result in permanent infertility, whilst NET-EN was considered ".... less strong and 'usually reversible' " p. 13 [4].

Age as a criterion for prescribing one or other IPC product is not supported by the literature, and some policy documents and publications specifically debunk the notion that IPCs should be restricted according to age. For instance, Lande recommends that:

"Providers may need to reassure clients and the public that injectables do not cause infertility but to note that women should expect a wait of some months after stopping injectables to become pregnant. Service policies based on a fear of infertility - in particular, age and parity restrictions - can be dropped p.7 [23].

Table 3: Side effects most frequently reported by DMPA and NET-EN users

\begin{tabular}{|c|c|c|c|}
\hline Side Effect & $\begin{array}{l}\text { DMPA (\%) } \\
(n=84)\end{array}$ & $\begin{array}{l}\text { NET-EN (\%) } \\
(n=95)\end{array}$ & P Value* \\
\hline \multicolumn{4}{|l|}{ Menstrual irregularities } \\
\hline Amenorrhoea $\#$ & 67.5 & 58.9 & 0.240 \\
\hline Spotting & 9.5 & 12.6 & 0.510 \\
\hline Heavy periods & 8.3 & 7.4 & 0.792 \\
\hline Irregular periods & 3.6 & 10.5 & 0.074 \\
\hline Longer periods & 2.4 & 4.2 & 0.497 \\
\hline Dysmenorrhoea & 1.2 & I.I & 0.930 \\
\hline Vaginal wetness & 22.6 & 14.7 & 0.175 \\
\hline Weight gain & 14.3 & 8.4 & 0.214 \\
\hline Loss of libido & 10.7 & 8.4 & 0.601 \\
\hline Dizziness & 10.7 & 6.3 & 0.289 \\
\hline Headache & 10.7 & 4.2 & 0.094 \\
\hline Nausea & 9.5 & 3.2 & 0.077 \\
\hline Vaginal discharge & 8.3 & 3.2 & 0.132 \\
\hline Vaginal discharge with odour & 7.1 & 3.2 & 0.223 \\
\hline
\end{tabular}

\footnotetext{
\# Includes thirty breastfeeding women. Although amenorrhoea was reported as a side effect of IPC use, it could have been lactational amenorrhoea

* Chi-square (I degree of freedom, significance tested at the $5 \%$ level)
} 
The second draft of the South African Department of Health's Draft National Framework \& Guidelines for Contraceptive Services explicitly states that:

"Young clients should not be prevented from using either DMPA or NET-EN because of their age." p.64 [24].

If one were to embrace the WHO promoted Essential Drugs concept [5] the decision about which IPC to supply should be made on cost since DMPA and NET-EN have comparable efficacy and safety profiles. Based on the cost analysis presented in this paper, DMPA should be the product selected. However, reducing contraceptive options flies in the face of progressive reproductive health policies which promote expansion of contraceptive choice. For instance, the WHO "is giving priority to improving access to high-quality care in family planning through a variety of strategies" p.2, and lists one of these strategies as "promoting the widest availability of different contraceptive methods so that people may select what is most appropriate to their needs and circumstances" p.2 [20]. The Programme of Action adopted at the International Conference on Population and Development held in Cairo in 1994 recommended that family planning programmes should "Recognize that appropriate methods for couples and individuals vary according to their age, parity, family-size preference and other factors, and ensure that women and men have information and access to the widest possible range of safe and effective family-planning methods in order to enable them to exercise free and informed choice" p.39/132 [25]. The Population Council's new approach to contraceptive introduction in developing countries involves an assessment of the context of contraceptive use in that country, on the basis of which "recommendations for upgrading contraceptive services - which could include introducing new methods, improving the utilisation of existing ones, and/or removing one or more from the method mix". p.1 [26].

The injectable contraceptive method is an important option in South Africa, since many women choose this method because its use does not require partner knowledge or consent [27]. The review of the literature shows that menstrual irregularities are reported to occur more often with DMPA than with NET-EN use. In cases where side effects such as amenorrhoea are particularly problematic with DMPA, NET-EN may be a good alternative. By providing NET-EN explicitly as a second-line option, the range of contraceptive products would be restricted, but not reduced.

\section{Conclusions and Recommendations}

Providing IPCs on the basis of age is not appropriate or cost effective. Training of health workers and counselling of clients to correct this misconception is clearly required. Where clients require immediate return to fertility upon discontinuing contraception, neither IPC preparation is ideal. Since DMPA is a cheaper option than NET-EN, health worker training about the rational use of injectable contraceptives should include consideration of the cost implications of prescribing one product over another. DMPA should be considered as the first option, but where DMPA is not well tolerated, NET-EN should be available as a second option. It is also recommended that a comparative systematic review of DMPA and NET-EN be undertaken. Based on the outcome of this review, consideration may be given to conducting a comparative clinical trial of NET-EN and DMPA when used by African women.

Consideration should be given to encouraging the registration of the combined injectable contraceptive in South Africa, which has a better side effect profile than the IPCs [28]. This would be an expensive option thus combined injectable contraceptives should only be provided where side effects with the IPCs are intolerable. A better contraceptive option, especially for young people, might however be the male or female condom with back up of emergency contraceptive pills to provide dual protection against unwanted pregnancy and HIV and other sexually transmitted infections.

\section{List of Abbreviations}

CYP couple years of protection

DMPA depot medroxyprogesterone acetate

EDL essential drugs list

KZN KwaZulu-Natal

IPCs injectable progestagen-only contraceptives

NET-EN norethisterone oenanthate

SAR South African Rand

WHO World Health Organisation

\section{Acknowledgements}

This study was supported by the Wellcome Trust (Grant Number 050522/ Z/97/Z). We thank Mr J van den Berg (Deputy Director: Procurement, National Department of Health) and Mr A Odendaal (Systems Manager, Coordinating Committee for Medical Procurement) for providing access to the data from the pharmaceutical depots and for the initial Pareto analysis.

\section{Competing Interests}

Have you in the past five years received reimbursements, fees, funding, or salary from an organisation that may in any way gain or lose financially from the publication of this paper? 
A travel grant was awarded to the main author of this paper in March 1997 by the manufacturer of the generic depot medroxyprogesterone acetate product - one of the drugs mentioned in this manuscript.

Do you hold any stocks or shares in an organisation that may in any way gain or lose financially from the publication of this paper? No.

Do you have any other financial competing interests? No.

Are there any non-financial competing interests you would like to declare in relation to this paper? No.

\section{References}

I. Department of Health of South Africa, Medical Research Council and Macro International : South Africa demographic and health survey 1998. Preliminary report. Pretoria, South Africa: Department of Health 1999

2. National Department of Health : South African standard treatment guidelines and essential drugs list for South Africa. Primary health care. South Africa: National Department of Health 1998

3. Beksinska ME, Rees VH, Nkoyane T, McIntyre JA: Compliance and use behaviour, an issue in injectable as well as oral contraceptive use? A study of injectable and oral contraceptive use in Johannesburg. British Journal of Family Planning 1998, 24(I):21-3

4. Wood K, Maepa J, Jewkes R: Adolescent sex and contraceptive experiences: perspectives of teenagers and clinic nurses in the Northern Province. Pretoria, South Africa: Medical Research Council 1997

5. Kanji N, Hardon A, Harnmeijer JW, Mandani M, Walt G: Drugs policy in developing countries. London: Zed Books 1992

6. Trussell J, Hatcher RA, Cates W, Stewart FH, Kost K: A guide to interpreting contraceptive efficacy studies. Obstetrics and Gynecology 1990, 76:558-67

7. WHO Special Programme of Research Development and Research Training in Human Reproduction, Task Force on Long-Acting Agents for the Regulation of Fertility : A multinational comparative clinical trial of long-acting injectable contraceptives: Norethisterone enanthate given in two dosage regimens and depotmedroxyprogesterone acetate. Final report. Contraception | 983, 28:|-2|

8. Kaunitz AM: Clinical use of depo-provera (medroxyprogesterone acetate) for contraception. A current perspective of scientific, clinical and social issues. In Proceedings of an International Symposium 19-20 November 1992. Edited by Zambrano D. Johannesburg, South Africa: Upjohn, 1992

9. Fraser IS, Weisberg E: Fertility following discontinuation of different methods of fertility control. Contraception 1982, 26:389415

10. Howard G, Blair M, Fotherby K, Elder MG, Bye P: Seven years clinical experience of the injectable contraceptive, norethisterone oenanthate. The British Journal of Family Planning 1985, I I:9-16

II. Bigrigg A, Evans M, Gbolade B, Newton J, Pollard L, Szarewski A, Thomas C, Walling M: Depo Provera. Position paper on clinical use, effectiveness and side effects. British Journal of Family Planning 1999, 25:69-76

12. Hatcher RA, Rinehart W, Blackburn R, Geller JS, Shelton JD: The essentials of contraceptive technology. Baltimore: Johns Hopkins University of Public Health, Population Information Program, 1997

13. Fraser IS: Menstrual changes associated with progestogenonly contraception. Acta Obstetricia et Gynecolologica Scandnavicia 1986, I34:21-7

14. Salem HT, Salah M, Aly MY, Thabet Al, Shaaban MM, Fathalla MF: Acceptability of injectable contraceptives in Assiut, Egypt. Contraception 1988, 38:697-710

15. World Health Organisation Expanded Programme of Research Development and Research Training in Human Reproduction : Multinational comparative clinical evaluation of two long-acting injectable contraceptive steroids: Norethisterone oenanthate and medroxyprogesterone acetate. 2. Bleeding patterns and side effects. Contraception 1978, 17:395-406

16. Fraser IS: $\mathbf{A}$ health perspective of hormonal contraceptives. Acta Obstetricia et Gynecologica Scandinavica 1986, 1 34:33-43

17. Kaunitz AM: Long-acting injectable contraception with depot medroxyprogesterone acetate. American Journal of Obstetrics and Gynecology 1994, I 70:1543-49
18. Shapiro S, Rosenberg L, Hoffman M, Truter H, Cooper D, Rao S, Dent D, Gudgeon A, van ZyIJ, Katzenellenbogen J, Baillie R: Risk of breast cancer in relation to the use of injectable progestogen contraceptives and combined estrogen/progestogen contraceptives. American Journal of Epidemiology 2000, I I I:396-403

19. Watts NB: Spinal bone density in women using depot medroxyprogesterone contraception Obstetrics and Gynecology 1998, 92:569-73

20. World Health Organisation : Improving access to quality care in family planning. Medical eligibility criteria for contraceptive use. Geneva: World Health Organisation. 1996

21. Quick JD, Rankin JR, Laing RO, O'Conner RW, Hogerzeil HV, Dukes MNG, Garnett A (eds): Managing drug supply. The selection, procurement, distribution and use of pharmaceuticals, $2 n d$ ed. West Hartford, Connecticut, USA: Kumarian Press, 1997

22. Janowitz $B$, Measham $D$, West $C$ : Issues in the financing of family planning services in Sub-Saharan Africa. USA: Family Health International, 1999

23. Lande RE: New era for injectables. Baltimore, Maryland, USA, Population Information Program, Center for Communication Programs, The Johns Hopkins School of Hygiene and Public Health. Population Reports 1995, Series $K$, No 5

24. Department of Health : National Framework \& Guidelines for Contraceptive Services. Republic of South Africa. Second Draft. Pretoria, South Africa: Department of Health, 1999

25. United Nations Population Information Network: Report of the International Conference on Population and Development, Cairo, 5-13 September 1994. New York, United Nations Publications, 1994 [http://www.undp.org/popin/icpd/conference/offeng/poa.html]

26. Population Council : Contraceptive introduction. Expanding contraceptive choice: Findings from Zambia. Population Briefs, Spring 1996, 2:2 [http://wwwpopcouncil.org/publications/popbriefs/ pb2\%282\%29\%5 F.html]

27. Kaufman CE: Reproductive control in South Africa. Policy Research Division Working Paper, No 97. New York: Population Council. 1997

28. Kaunitz AM, Garceau RJ, Cromie MA: Comparative safety, efficacy, and cycle control of Lunelle ${ }^{\mathrm{TM}}$ monthly contraceptive injection (medroxyprogesterone acetate and estradiol cypionate injectable suspension) and Ortho-Novum 7/7/7 oral contraceptive (norethindrone/ethinyl estradiol triphasic). Lunelle Study Group. 1999, 60:179-87

\section{Pre-publication history}

The pre-publication history for this paper can be accessed here:

http://www.biomedcentral.com/content/backmatter/ 1472-6963-1-4-b1.pdf

\section{Publish with BioMedcentral and every} scientist can read your work free of charge

"BioMedcentral will be the most significant development for disseminating the results of biomedical research in our lifetime." Paul Nurse, Director-General, Imperial Cancer Research Fund

Publish with BMc and your research papers will be:

- available free of charge to the entire biomedical community

- peer reviewed and published immediately upon acceptance

- cited in PubMed and archived on PubMed Central

- yours - you keep the copyright

Submit your manuscript here:

http://www.biomedcentral.com/manuscript 